Reprod. Nutr. Dévelop., 1980, 20 (3 B), 759-770.

\title{
Le comportement parental des Dermaptères
}

\author{
par M. VANCASSEL, Maryvonne FORASTE
}

ERA 489 CNRS, Laboratoire d'Ethologie

Université de Rennes, Campus de Beaulieu, 35042 Rennes Cedex.

\section{Summary. Parental relations in Dermaptera.}

Parent-young relations are far from being unvarying and simple, even within a group as homogeneous and modest in number (1000 actual species) as Dermaptera.

Care of the eggs by a female and the contacts she maintains with her larvae are neither established nor developed in an automatic way, but are amenable to important regulations in which behaviour itself is implied.

The presence of larvae also has an important effect on the later development of the female's behaviour. Relationships can be established between known inter and intraspecific differences and between.

- the ovarian organization and activity of each species or population, on the one hand and

- the living conditions adopted by each species or population (Labidura riparia, Anechura bipunctata, Forficula auricularia) on the other.

Collecting all the eco-etho and physiological data can be useful for understanding the development of Dermapteran maternal behaviour.

\section{1. - Introduction.}

Le comportement parental des Dermaptères s'organise toujours en deux phases distinctes :

- Les soins prodigués aux œufs par la femelle depuis la ponte jusqu'à l'éclosion caractérisent la première phase. Exceptés certains insectes sociaux, ces contacts directs entre la femelle et sa ponte ne semblent pas avoir d'équivalent dans aucun autre groupe d'insectes où les œufs sont simplement gardés par la pondeuse. La valeur biologique des soins repose sur le fait que les œufs des Dermaptères, sensibles aux micro-organismes du sol, avortent lorsqu'ils n'en sont pas régulièrement débarrassés (Thiagarajan, 1939 ; Lhoste, 1941 ; Knabke et Grigarick, 1971 ; Caussanel, 1975). Les soins consistent en un brossage du chorion exercé par la femelle grâce aux soies de certaines parties de ses pièces buccales (lacinia).

- La deuxième phase parentale commence à l'éclosion. Elle est marquée par les contacts que la femelle entretient avec ses larves. Cette phase se termine soit par la dispersion des larves, soit par l'abandon du terrier par la femelle, soit encore par la mort de celle-ci. 
Si la première période de la séquence parentale est très uniforme d'une espèce à l'autre, à sa durée près, la deuxième varie beaucoup dans son organisation même.

Le but de l'étude comparative présentée ici est d'essayer de comprendre comment ces différences d'organisation du comportement parental ont pu se constituer et ce qu'elles signifient.

\section{2. - Les différentes stratégies de reproduction des Dermaptères.}

L'observation directe de 10 espèces différentes appartenant à diverses familles de Dermaptères et l'analyse de la littérature qui comporte des indications sur une vingtaine d'espèces (1 000 à 1200 espèces sont actuellement recensées pour l'ensemble du groupe) permettent de décrire trois types de stratégies de reproduction différents représentés par les trois espèces suivantes:

1. - Labidura riparia (Labiduridae) (origine Camargue, Bouches-du-Rhône).

2. - Anechura bipunctata (Forficulidae) (origine Briançon, Hautes-Alpes).

3. - Forficula auricularia (Forficulidae) (Origine Pacé, llle-et-Vilaine).

a) Dans le premier type de stratégie chaque femelle produit plusieurs pontes et après chaque éclosion les larves se dispersent rapidement (avant la première mue larvaire). Entre deux phases parentales la femelle s'alimente et une vitellogenèse a lieu. Dans le cas de $L$. riparia, espèce prédatrice, la femelle approvisionne son terrier en nourriture entre l'ouverture du terrier ( $3 \mathrm{e}$ ou $4^{\mathrm{e}}$ jour après l'éclosion) et la fin de la dispersion des larves (6e ou $7 \mathrm{e}$ jour après l'éclosion) (fig. 1).

A. bip.

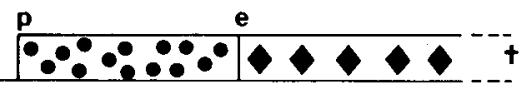

L. rip.

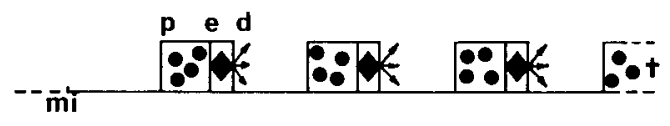

F. aur.

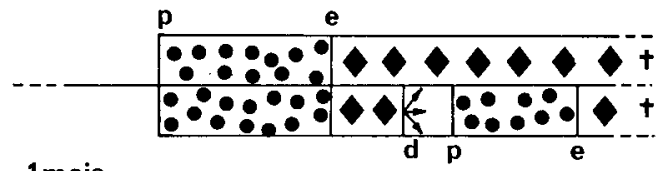

1 mois $=$

FIG. 1. - Stratégies de reproduction chez les Dermaptères. Trait simple - : phase non parentale ; - : phase de soins des œufs; : phase de groupement avec les larves; $\dagger$ : mort de la femelle ; d et $\&$ : dispersion ; e : éclosion ; mi : mue imaginale de la femelle ; $p$ : ponte.

b) Le deuxième type de stratégie, adopté par Anechura bipunctata, se caractérise par une seule ponte par femelle. Après l'éclosion les larves restent au terrier. La femelle approvisionne celui-ci en nourriture. La très longue phase de contacts entre la femelle ef les larves qui s'ensuit n'est interrompue que par la mort de la femelle (fig. 1). 
c) Le dernier type de stratégie est intermédiaire des deux précédents. Ici certaines femelles ne produisent qu'une seule ponte et entretiennent des contacts avec leurs larves pendanf une longue période (jusqu'à la mort de la pondeuse comme chez Anechura bipunctata). Les autres femelles s'isolent de leurs larves 3 ou 4 semaines après l'éclosion et produisent une deuxième ponte. Chez $F$. auricularia la femelle peut apporter de la nourriture au terrier mais les larves quittent aussi celui-ci pendant la phase d'activité nocturne et s'alimentent seules à l'extérieur. Le retour au terrier, à la fin de chaque période d'alimentation, est général mais une dispersion larvaire plus ou moins importante se produit aussi toujours à cette occasion.

\section{3. - L'organisation ovarienne des Dermaptères.}

Ces stratégies de reproduction reposent d'abord sur des structures et des fonctionnements ovariens différents.

a) Chez L. riparia chaque ovaire ne compte que 5 ovarioles mais chacune de celles-ci possède un germarium fonctionnel durant toutes la vie imaginale de la femelle. A chaque vitellogenèse chaque ovariole fournit 8 à 10 œufs. Tous les œufs mûrs sont pondus ensemble au début de la phase parentale (fig. 2).

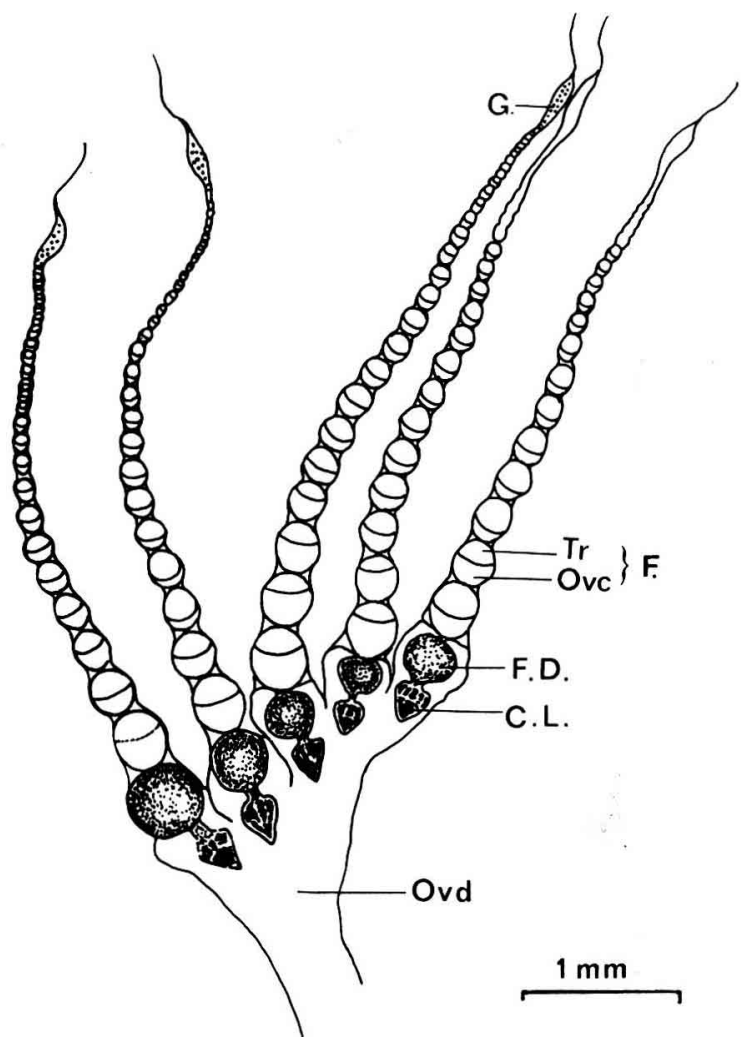

FIG. 2. - Ovaire de Labidura riparia (après une ponte). C.L. : corpus luteum ; F. : follicule ; F.D. : follicule en dégénérescence ; G. : germarium ; Ovc : ovocyte ; Ovd : oviducte ; Tr : trophocyte. 
b) L'ovaire d'Anechura bipunctata est organisé différemment : il comple de nombreuses ovarioles mais chacune ne possède qu'un ovocyle. Tous les œufs mûrs sont pondus ensemble de telle sorte qu'après cette ponte unique les ovaires se réduisent aux gaines des ovarioles ef oviductes. Après la ponte les femelles d'Anechura sont des castrats fonctionnels (fig. 3).
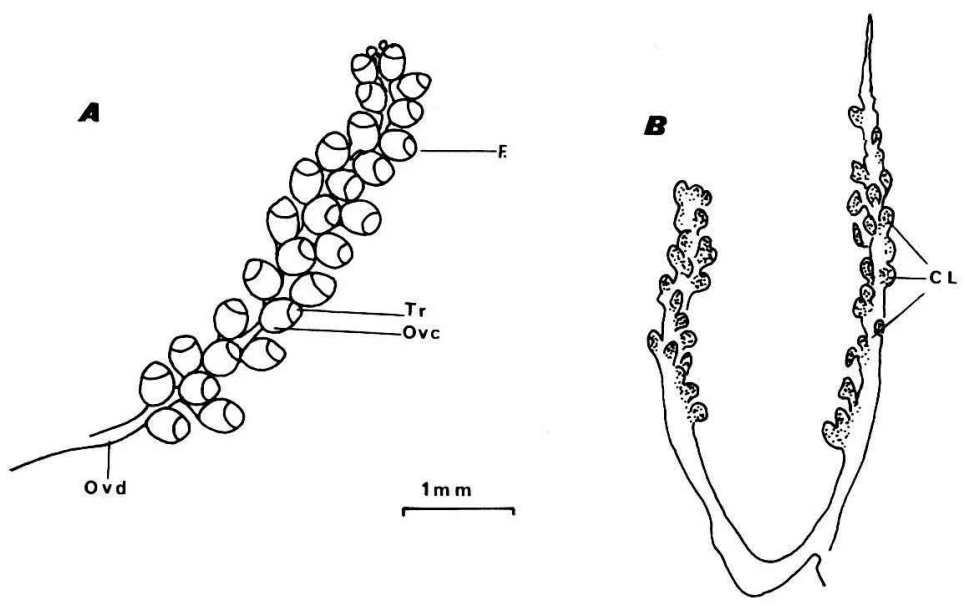

FIG. 3. - Ovaire d'Anechura bipunctata.

$A$ : début de vitellogenèse; $B$ : après ponte. (Légende voir fig. 2).

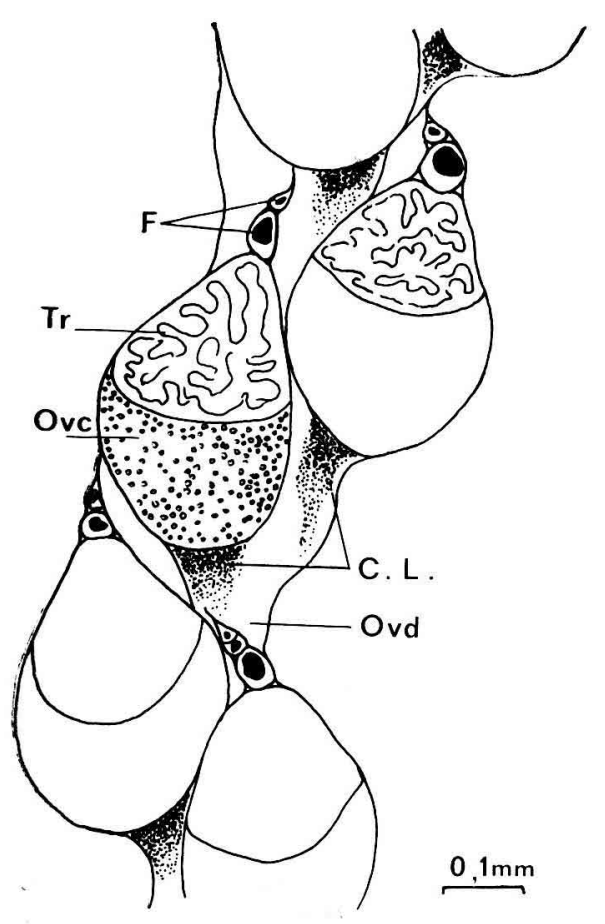

FIG. 4. - Ovaire de Forficula auricularia (après:la première [ponte). (Légende voir fig. 2.) 
c) Le plan d'organisation de l'ovaire de $F$. auricularia est encore intermédiaire : ovarioles nombreuses, sans germarium mais chacune comptant plusieurs ovocytes. A chaque vitellogenèse un seul ovocyte, organisé dans un follicule, se développe dans chaque ovariole. Tous les œufs mûrs sont ici aussi pondus en une seule fois (fig. 4).

\section{4. - La régulation des aptitudes parentales.}

Il existe aussi entre les espèces des différences importantes dans la régulation des aptitudes parentales de la femelle. Parmi celles-ci nous avons choisi d'évoquer le maintien de l'aptitude de la femelle à accepter les contacts des larves parce qu'il est manifestement en rapport avec le fonctionnement ovarien.

a) Chez L. riparia les femelles possèdent deux types de réaction à l'égard des larves : en phase parentale elles acceptent leurs contacts; en phase non parentale elles les capturent et les mangent.

On peut prolonger la phase parentale des femelles en les soumettant au jeûne En même temps on retarde la vitellogenèse qui suit cette phase parentale.

Mais la dispersion des larves joue aussi un rôle dans l'extinction habituellement rapide de cette aptitude de la femelle à accepter leur contact.

Au laboratoire on mime une dispersion plus ou moins importante des larves en installant des femelles et leur ponte dans des enceintes de mêmes dimensions ; certaines femelles sont laissées avec tous les œufs $(\geqslant 50)$ puis leurs larves et obtiennent de nombreux contacts les jours suivants; les autres laissées avec seulement 15 à 20 œufs la veille de l'éclosion obtiennent beaucoup moins de contacts avec leurs larves. Toutes les femelles et larves sont maintenues en jeûne. Les femelles du premier lot ont des contacts positifs avec leurs larves beaucoup plus longtemps que celles à qui on a laissé un petit groupe de larves (dispersion plus importante) et qui passent plus vite à la prédation (fig. 5) (Vancassel, 1977).

FIG. 5. - Influence du nombre des contacts femelle-larves sur le maintien de l'aptitude de la femelle à accepter des larves. Comparaison jour après jour du nombre total de femelles acceptant les larves dans les lots A : nombre normal de larves + jeône et B : nombre réduit de larves + jeûne.

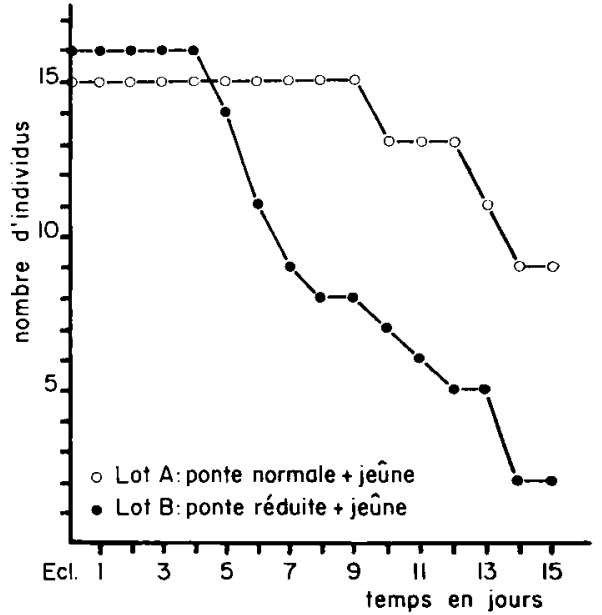


b) Chez $A$. bipunctata la situation est complètement différente. La femelle ne capture jamais les larves ef de plus après la ponte elle reste indéfiniment apte à soigner des œufs et à accepter le contact des larves.

Plusieurs semaines d'isolement après l'éclosion n'ont aucune influence : lorsque la femelle est replacée avec des jeunes, elle réorganise un terrier et reprend son comportement parental d'approvisionnement, indépendamment du stade de développement des jeunes.

c) II n'en va pas de même avec $F$. auricularia chez qui l'isolement après l'éclosion entraîne la disparition de l'aptitude à soigner les œufs. Comme cette espèce ne pratique pas de prédation sur les larves et que ces dernières se dispersent spontanément à chaque phase d'alimentation il devient difficile de juger du devenir de l'aptitude de la femelle à garder le contact avec ses jeunes. Néanmoins Lamb (1976) établit que les femelles prêtes à produire une deuxième ponte abandonnent leur terrier et rompent leur lien avec leurs larves. De ce fait l'observation d'une deuxième ponte chez une femelle implique la disparition préalable de cette aptitude parentale envers les larves.

Lorsque nous isolons des femelles après l'éclosion de leur première ponte et que nous les comparons avec des femelles laissées au contact de leurs jeunes nous observons que les premières produisent très souvent une deuxième ponte tandis que les autres n'en produisent que très rarement (tabl. 1). A la dissection ces dernières montrent des ovaires peu ou pas développés au moment où les autres ont déjà déposé leur deuxième lot d'œufs (30 jours environ après l'éclosion).

TABLEAU 1

Influence de la présence des larves sur la répétition de la pante des femelles de Forficula auricularia (Pacé). (Nourriture $=$ Carotte)

\begin{tabular}{lccc}
\hline & $\begin{array}{c}2^{\mathrm{e}} \text { ponte } \\
\text { observée }\end{array}$ & $\begin{array}{c}2^{\mathrm{e}} \text { ponte } \\
\text { non observée }\end{array}$ & Total \\
\hline $\begin{array}{l}\text { Femelles isolées après l'éclosion de la } \\
\text { fre ponte } \ldots \ldots \ldots \ldots \ldots \ldots \ldots \ldots \ldots\end{array}$ & 11 & 6 & 17 \\
\hline Femelles + Larves............... & 0 & 14 & 14 \\
\hline
\end{tabular}

Nous en concluons que la présence des larves a pour effet d'entretenir l'aptitude de la femelle à garder le contact et, au minimum, de ralentir considérablement la vitellogenèse de celle-ci.

\section{5. - L'in fluence des conditions climatiques.}

Pour terminer il nous faut évoquer la relation qui semble exister entre la stratégie de reproduction adoptée par chaque espèce et les conditions climatiques subies par celle-ci.

La population de L. riparia que nous étudions vit en Camargue, c'est-à-dire sous un climat méditerranéen ; celle d'A. bipunctata est établie dans les Alpes (de 1800 à 
$2300 \mathrm{~m}$ d'altitude) ; enfin celle de F. auricularia vit en Ille-et-Vilaine et subit un climat tempéré océanique.

Si l'on tient compte des vitesses de développement ovarien et embryonnaire de chaque espèce (qui varient selon la température) et qu'on les confronfe aux moyennes des températures mensuelles subies par elles dans leur milieu on constate le fait suivant : même si l'on suppose qu'une dispersion rapide des larves se produit dans tous les cas après l'éclosion, seule l'espèce L. riparia peut produire 3 pontes dans son milieu ; F. auricularia peut en produire deux et Anechura bipunctata un peu plus d'une (fig. 6).

FIG. 6. - Courbes des températures moyennes mensuelles : en Camargue (Bouches-du-Rhône) : $\mathrm{C}$; St-Jacques-de-la-Lande (Ille-et-Vilaine) : St $\mathrm{J}$; Font-Romeu (Pyr. orientales) : Ft-R. Sur chaque courbe on a porté à titre indicatif la durée du développement embryonnaire ( $(\square)$ et la durée de la vitellogenèse $(\square)$ de $L$. riparia (température moyenne extérieure de $20^{\circ} \mathrm{C}$ ) el de F. auricularia $\left(16^{\circ} \mathrm{C}\right.$ ef $\left.10^{\circ} \mathrm{C}\right)$.

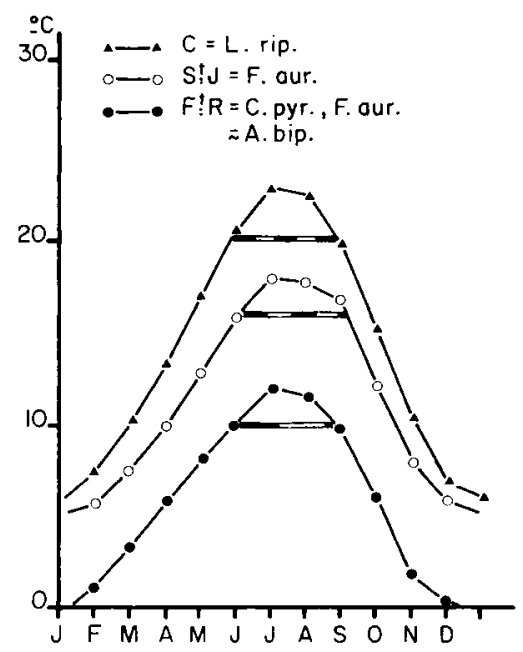

Nous retenons donc l'hypothèse d'une relation entre la stratégie de production développée par une espèce et les conditions climatiques subies par cette espèce et nous la formulons comme suit : A une longue période favorable (à la vitellogenèse et au développement embryonnaire et larvaire) correspond une augmentation du nombre des pontes associée à des contacts femelle-larves de courte durée ; tandis qu'à une période favorable courte correspond une réduction du nombre des pontes associée à des contacts femelle-larves de longue durée?

Actuellement cette hypothèse paraît satisfaite pour l'ensemble des espèces dont le comportement parental a éłé décrit.

Nous avons pu la vérifier par des observations de terrain et l'élévage au laboratoire pour les espèces suivantes :

a) Espèces du type Labidura : Euborellia moesta ef Anisolabis mauritanica (espèces méditerranéennes) et Spendax sp. et Euchenomus sp. (espèces tropicales).

b) Espèces du type Anechura : Chelidura pyrenaïca (espèce pyrénéenne établie au dessus de $1500 \mathrm{~m}$ dans la région de Font-Romeu, Pyrénées orientales) identique en tous points à $A$. bipunctata mais pour laquelle cependant le mode de dispersion des larves n'est pas établi.

c) Espèces du type Forficula : $F$. lesnei et $F$. decipiens (espèces de régions tempérées, formes observées en Ille-et-Vilaine). 
La rencontre d'une population pyrénéenne de $F$. auricularia établie à $1600 \mathrm{~m}$ d'altitude (où elle vit en sympatrie avec $C$. pyrenaíca) nous a donné aussi l'occasion de comparer deux populations de la même espèce vivant dans deux situations climatiques différentes.

Cette étude a permis d'établir une différence significative dans l'aptitude des femelles de ces deux populations à produire deux pontes successives.

Pour mesurer cette capacité à produire deux pontes on tient comple des résultats précédents en isolant toutes les femelles après l'éclosion de leur première ponte : 58 p. 100 des femelles de la population pyrénéenne (Font-Romeu) produisent une deuxième ponte contre 83 p. 100 des femelles de la population de référence (Pacé) (tabl. 2). Cette différence se traduit au niveau de l'organisation ovarienne des femelles: celles de la population Font-Romeu ont moins d'ovocytes par ovariole que celles de la population de référence (tabl. 3). Cette observation va bien dans le sens de l'hypothèse proposée.

\section{TABLEAU 2}

Nombre de pontes produit par les femelles de F. auricularia (Comparaison Font-Romeu Pacé). (Isolement à l'éclosion de la première ponte et nourriture à base de pollen)

\begin{tabular}{lccc}
\hline & 1 ponte & 2 pontes & \\
\hline Pacé $\ldots \ldots \ldots \ldots$ & 9 & 47 & 83 p. 100 \\
\hline Font-Romeu $\ldots$ & 9 & 13 & 58 p. 100 \\
\hline$; \alpha=0,05)$.
\end{tabular}

TABLEAU 3

Nombre de trophocytes par ovariole (Comparaison Font-Romeu Pacé)

\begin{tabular}{lccc}
\hline & $\begin{array}{c}\text { Nombre } \\
\text { d'ovarioles }\end{array}$ & $\begin{array}{c}\text { Moyenne } \\
\text { Troph./ovar. }\end{array}$ & $\mathrm{S}$ \\
\hline Font-Romeu & 229 & 3,12 & 1,12 \\
\hline Pacé $\ldots \ldots \ldots$ & 185 & 5,27 & 1,42 \\
\hline
\end{tabular}

(Test $T$ de student, $P \leqslant 0.001$ ).

II faut ajouter à cela que l'observation de la population de Font-Romeu permet de penser que les femelles de cette population ne produisent effectivement qu'une seule ponte : en effet durant la deuxième moitié du mois d'août cette population ne compte que des larves de dernier stade et des jeunes adultes. Si deux pontes étaient produites on devrait trouver au moment de la mue imaginale des individus de la première cohorte (éclosion en avril), des stades larvaires plus jeunes correspondant à la deuxième cohorte comme c'est le cas pour la population de Pacé. 
A l'évidence, l'organisation ovarienne particulière des femelles de Font-Romeu ne peut rendre compte à elle seule de cette absence d'une deuxième cohorte puisque plus de la moitié des femelles de la population Font-Romeu sont capables de pondre deux fois.

L'effet de la présence des larves issues de la première ponte peut être à l'origine d'une disparition complète de la deuxième ponte à condition toutefois de vérifier que, dans les conditions de Font-Romeu, la dispersion des larves est elle-même atténuée, voire supprimée.

\section{6. - Conclusions et discussion.}

La singularité du comportement parental des Dermaptères, sa présence probable dans tout l'ordre en font un système comportemental unique. La forme qu'il revêt chez chaque espèce ou chaque population constitue un état particulier de ce système et notre démarche générale consiste à étudier les différents facteurs et conditions qui permettent la différenciation de ces états.

L'organisation du système ovarien pèse énormément sur la stratégie de reproduction adoptée par chaque espèce, en fixant les possibilités extrêmes de celle-ci (nombre de pontes).

D'autre part les conditions climatiques subies, ou adoptées, par chaque espèce ou population constituent un facteur limitant important puisqu'elles déterminent la durée favorable au développement ovarien, embryonnaire et larvaire de chaque espèce.

Le cas particulier de $F$. auricularia fait intervenir de manière efficace un troisième facteur, d'ordre comportemental : l'effet de la présence des larves sur le développement de la femelle (évolution de ses aptitudes parentales ef vitellogenèse).

Si l'on accepte l'idée que l'efficacité de ce facteur est elle-même conditionnée par des facteurs écologiques (entraînant une dispersion plus ou moins rapide des larves) et anatomo-physiologique (organisation et fonctionnement ovariens), toutes choses probables sinon encore démontrées, on réalise du même coup la mise en relation des trois aspects physiologique, écologique et comportemental que nous avons abordés.

Concernant l'effet de la présence des larves sur l'état motivationnel des femelles cette problématique permettrait de comprendre à la fois :

- la présence de cet effet, sans aucune efficacité, chez les espèces présentant une organisation et un fonctionnement ovariens du type L. riparia ;

- la disparition pure ef simple de cet effet chez les espèces présentant une organisation ovarienne du type $A$. bipunctata où les femelles ne sont plus que des castrats fonctionnels après la ponte.

Ceci constifue un encouragement pour un développement et un approfondissement de cette synthèse éco-étho-physiologique.

Qu'en est-il des différents états du système comportemental dont nous avons entrepris l'analyse? Nous croyons pouvoir dire dès maintenant que selon les circonstances ce système s'est modifié aussi bien dans le sens : 1. - d'une multiplication des phases parentales avec des contacts femelle-larves de courte durée, que dans le sens ; 2 . - 
d'une réduction du nombre des phases parentales avec possibilité de développement d'une longue période de contacts entre la femelle at ses larves.

Pour le premier cas reprenons l'exemple de Labidura riparia : les femelles de cette espèce présentent deux particularités. La première consiste dans l'approvisionnement du terrier en nourriture, fugace mais constant, réalisé au cours de la dispersion des larves. La deuxième est la sensibilité des femelles à la présence expérimentale des larves, grâce à laquelle la réaction positive aux larves peut être prolongée.

Il est difficile de comprendre la différenciation de ces deux caractéristiques car la dispersion rapide des larves atténue, ou même supprime, toute leur valeur de survie possible. Cette difficulté est renforcée par le fait que la famille des Labiduridae est classiquement considérée comme primitive parmi les Dermaptères.

Or récemment Popham (1965) s'appuyant non plus seulement sur l'organisation des genitalia mais sur plusieurs systèmes de critères morphologiques a aftribué une position intermédiaire à la famille des Labiduridae.

En fait le comportement d'approvisionnement par la femelle est bien adapté chez les espèces omnivores chez qui il a pu se développer originellement (Pygidicranidae et Diplatyadae) et chez les espèces omnivores où il s'est maintenu, voir développé, secondairement (Forficulidae). Par contre, chez les espèces carnivores et prédatrices ce comportement serait inadapté (retour rapide de la prédation des larves par la femelle) et sans valeur de survie. Chez Labidura riparia par exemple il ne serait plus qu'un vestige.

Le même raisonnement peut être invoqué pour la sensibilité de la femelle vis-àvis des larves.

Mais simultanément il faut conclure que l'état du système comportemental des Dermaptères, représenté par Labidura riparia, constitue une étape dans le sens d'une augmentation du nombre de pontes et d'une réduction des contacts entre la femelle ef les larves. La fendance opposée peut être reconnue chez l'espèce Forficula auricularia.

Dans nos régions cette espèce est présente partoư sauf dans les régions montagneuses au-delà d'une certaine altitude (1600-2000 m). Cette distribution en nappe suggère que les populations trouvées en altitude constituent les avancées extrêmes de cette espèce vers de nouveaux milieux ; avancées probablement réalisées en Europe dans le contexte récent du recul des dernières glaciations. S'il en est ainsi, la population de Font-Romeu par exemple constituerait une forme récente issue de formes de plaines. Nous aurions donc là un exemple de dérive du système comportemental des Dermaptères dans le sens d'une réduction du nombre de pontes pouvant être associé à l'allongement des contacts entre la femelle et ses larves.

Grâce à leur comportement parental les Dermaptères ont droit au statut d'insectes sub-sociaux. On sait que chez les Insectes l'eu-socialité est atteinte sous les conditions suivantes : - soins à la progéniture prodigués par plusieurs individus ; - existence de castes avec division des activités ; - superposition des générations.

D'autre part les spécialistes envisagent deux voies d'accès possible à cet état d'eu-socialité : la voie parasociale où la société est initiée par l'association d'au moins deux femelles de même génération ; la voie sub-sociale où la société se constitue à partir d'un individu femelle et sa progéniture. Ces deux voies n'étant pas nécessairement exclusives l'une de l'autre. 
Quelle est la situation des Dermaptères par un rapport à cette question ?

Chez toutes les espèces connues, à l'exception d'Anechura bipunctata, la femelle, à partir de la ponte, défend son terrier contre tout congenère y compris une autre femelle en phase parentale. Chez A. bipunctata, par contre, deux femelles ayant pondu dans deux terriers voisins peuvent se rencontrer sans exprimer ces réactions : les deux individus rassemblent alors leurs œufs qu'elles soignent indistinctement puis alimentent leurs larves ensemble. Par cette communauté, cette espèce se hisse alors au stade qualifié de quasisocial ; mais les femelles ne pouvant produire qu'une seule ponte les choses ne peuvent aller plus loin.

Pour toutes les autres espèces qui ne tolèrent pas la présence d'une autre femelle, seule la voie sub-sociale peut être envisagée pour un accès à l'eu-socialité. Ici la relation observée entre des pontes multiples et une phase de contacts femelle-larves constitue un obstacle majeur à cette progression vers un état social plus élaboré.

II semble ainsi que les Dermaptères se voient couper l'accès à l'eu-socialité plus par l'organisation de leur système comportemental parental que par leurs aptitudes de communications interindividuelles.

Toutes les données précédentes concernent davantage la fonction du lien parental que sa nafure et il paraît quand même important d'aborder ce dernier aspect dans un colloque dont le thème est la «relation parentale » depuis l'invertébré jusqu'à l'homme. En fait la nature de cette relation parentale est encore mal connue chez les Dermaptères car traditionnellement les auteurs se sont plus attachés à l'étude de la femelle qu'à celle du groupe femelle-larves (Fulton, 1924 ; Weyrauch, 1929).

Depuis quelques mois Bourez a entrepris d'étudier plus particulièrement les relations interindividuelles chez $F$. auricularia. Il montre (communication personnelle) que le groupe femelle-larves se constitue à partir d'une attraction des larves par la femelle. Cette attraction, qui se réalise à distance, est exercée par toute femelle engagée dans une phase parentale. Elle est vraisemblablement d'ordre chimique. D'autre part, en présence de la femelle, les larves sollicitent celle-ci et obtiennent d'elle des contacts buccaux, apparemment identiques à ceux que les larves échangent aussi entre elles (Esslen, 1972). Ces contacts maintiennent la femelle dans son état parental, ce qui se traduit comme nous l'avons vu par le ralentissement, voir le blocage, du développement ovarien de celle-ci : en effet placée en contact olfactif mais non physique avec ses larves une femelle développe une deuxième ponte comme une femelle isolée.

A l'inverse, l'influence de la présence de la femelle sur le développement des larves est beaucoup plus difficile à cerner : lorsqu'elles se dispersent naturellement les larves de $F$. auricularia perdent l'abri que constitue le terrier de ponte et s'exposent aux nécessités d'un développement solitaire, mais par ailleurs ce développement ne paraît en lui-même nullement affecté.

Ce que nous connaissons de l'espèce $A$. bipunctata paraît encore plus simple : la femelle, qu'elle reste ou non avec ses larves après l'éclosion, garde intactes toutes ses aptitudes parentales, ceci vraisemblablement en relation avec une activité ovarienne nulle. Quant aux larves, elles tirent de la présence de la femelle la protection du terrier ef l'approvisionnement en nourriture mais apparemment rien d'autre.

En fait, la liaison parentale chez les Dermaptères, au moins telle qu'elle a pu être étudiée jusqu'ici, se place au niveau biosocial défini par Schneirla dans les années 40 
et 50 par contraste avec un niveau psychosocial où la liaison parentale intègre, entre autres, un processus ontogénétique de socialisation du jeune.

Cette distinction de niveaux différents a été reprise et modulée depuis, notamment par Tavolga (1970) sur le thème de la communication. Cependant, même sous sa forme primitive elle reste essentielle car, lorsque s'ouvre une discussion générale sur le comportement parental, elle évite de se laisser abuser par le contenu analogique de ce terme.

Colloque « La relation parentale " Nouzilly, France, juin 1979.

\section{Références}

CAUSSANEL C., 1975. Cycles reproducteurs de la femelle de Labidura riparia (Insecte Dermaptère) et leurs contrôles neuroendocrines. Thèse d'Etat, Paris VI.

ESSLEN J., 1972. Gegenseitiges Belecken beim ohrwuren Forficula auricularia L. Z. Tierpsychol., 30, 449-450.

FULTON B. B., 1924. Some habits of Earwigs. Ann. Ent. Soc. Amer., 17, 357-367.

KNABKE J. G., GRIGARIK A. A., 1971. Biology of the African earwig, Euborellia cincticollis in California and comparative notes on Euborellia annulipes. Hilga, 41, 7, 157-194.

LAMB R. J., 1976. Dispersal by nesting earwigs, Forficula auricularia (Dermaptera : Forficulidae). Can. ent., 108, 213-216.

LHOSTE J., 1941. Importance relative des soins maternels chez F. auricularia. C. R. Soc. Biol. Paris, 135, 499-500.

POPHAM E. J., 1965. The functional morphology of the reproductive organs of the common earwig (Forficula auricularia) and other Dermaptera with reference to the natural classification of the order. J. Zool., 146, 1-43.

SCHNEIRLA T. C., 1946. Problems in the biospychology of social organizations. J. Abnor. Soc. Psychol., 41, 385-402.

SCHNEIRLA T. C., 1951. The " levels " concept in the study of social organization in animals, 83-120. In SHERIF M., ROHER J. N. Social psychology of the crossroads. Harper New York.

SCHNEIRLA T. C., ROSENBLATT, J. C., 1961. Behavioral organization and genesis of the social bond in insects and mammals. Am. J. Orthopsychiat., 31, 223-253.

TAVOLGA W. N., 1970. Levels of interaction in animal communication, 281-302. In ARONSON, L. TOBACH, E. LEHRMAN, D. ROSENBLATT, J., Development and evolution of behavior (Essays in memory of T. C. SCHNEIRLA), Freeman and $\mathrm{C}^{\circ}$, San Francisco.

THIAGARAJAN K. B., 1939. The habits of the common earwig of annamalainagar Euborellia stali. J. Bombay. Nat. Hist. Soc., 40, 1 et 2, 721-723.

VANCASSEL M., 1977. Le développement du cycle parental de Labidura riparia. Biol. Comport., 2, 51 64.

WEYRAUCH W. K., 1929. Experimentelle Analyse der Brutpflege des Ohrwurmes Forficula auricularia L. Biol. Zentralbl., 49, 543-558. 